\title{
Dynamics of a mechanistically derived stoichiometric producer-grazer model
}

\author{
Hao Wang ${ }^{a *}$, Yang Kuang ${ }^{\mathrm{a}}$ and Irakli Loladze ${ }^{\mathrm{b}}$ \\ ${ }^{a}$ Department of Mathematics, Arizona State University, Tempe AZ, USA; ${ }^{b}$ Department of \\ Mathematics, University of Nebraska - Lincoln, Lincoln, NE, USA
}

(Received 24 July 2007; final revision received 13 October 2007)

\begin{abstract}
One of the simplest predator-prey models that tracks the quantity and the quality of prey is the one proposed by [I. Loladze, Y. Kuang, and J.J. Elser, Stoichiometry in producer-grazer systems: Linking energy flow with element cycling, Bull. Math. Biol. 62 (2000) pp. 1137-1162.] (LKE model). In it, the ratio of two essential chemical elements, carbon to phosphorus, C:P, represents prey quality. However, that model does not explicitly track P neither in the prey nor in the media that supports the prey. Here, we extend the LKE model by mechanistically deriving and accounting for P in both the prey and the media. Bifurcation diagrams and simulations show that our model behaves similarly to the LKE model. However, in the intermediate range of the carrying capacity, especially near the homoclinic bifurcation point for the carrying capacity, quantitative behaviour of our model is different. We analyze positive invariant region and stability of boundary steady states. We show that as the uptake rate of $\mathrm{P}$ by producer becomes infinite, LKE models become the limiting case of our model. Furthermore, our model can be readily extended to multiple producers and consumers.
\end{abstract}

Keywords: stoichiometry; Droop model; logistic equation; producer-grazer model; phosphorus uptake

AMS Subject Classification: 92D25; 34C60

\section{Introduction}

Ecological stoichiometry is the study of the balance of energy (carbon) and multiple chemical elements (phosphorus, nitrogen, etc.) in ecological interactions [11]. All organisms are composed of these elements. The relative abundances of them vary considerably among species and across trophic levels; hence, this chemical heterogeneity among species can have significant effects on food web dynamics. From the stoichiometric point of view, both food quantity and food quality need to be implicitly or explicitly modeled in producer-consumer interactions, since it is observed that the plant quality as well as its quantity can dramatically affect the growth rate of herbivorous grazers and may even lead to their extinction. On the other hand, plants are indeed limited by nutrient availability [3], and herbivores are more nutrient-rich organisms than plants, inducing food quality constraints. Stoichiometric theory has strong potential for both quantitative and qualitative improvements in the predictive power of population ecology $[2,10]$.

*Corresponding author. Email: wanghao@math.gatech.edu

ISSN 1751-3758 print/ISSN 1751-3766 online

(C) 2008 Taylor \& Francis

DOI: $10.1080 / 17513750701769881$

http://www.informaworld.com 
The rapidly growing empirical study of ecological stoichiometry facilitates a variety of stoichiometric population models in recent years $[1,4,5,7,8,9]$. Simple phenomenological twodimensional producer-consumer models, such as [8,9], are mathematically tractable but some of its simplifying assumptions may not hold in reality. Kuang et al. [7] gave a reasonable interpretation of the Loladze, Kuang and Elser (LKE) model using the Droop equation for the plant growth. This paper is to formulate the stoichiometric plant-herbivore model more mechanistically by explicitly modelling $\mathrm{P}$ content in the prey and in the media, thus relaxing a main assumption in the LKE model. Our model is a system of four autonomous ordinary differential equations, which can be reduced to a three-dimensional system.

To compare our model formulation with the LKE model, it is convenient to recall the main assumptions used in the LKE model.

(A1) The total mass of phosphorus in the entire system is fixed.

(A2) Phosphorus to carbon ratio $(P: C)$ in the plant varies, but it never falls below a minimum $q$ $(\mathrm{mg} P / \mathrm{mgC})$; the herbivore maintains a constant $P: C$ ratio, denoted by $\theta(\mathrm{mg} P / m g C)$.

(A3) Phosphorus in the system is divided into two pools: phosphorus in the herbivore and phosphorus in the plant.

\section{Model formulation}

Let $x$ be the density of carbon content in the producer, $p$ be the density of phosphorus content in the producer, $y$ be the density of carbon content in the grazer and $P$ be the density of free phosphorus in the media. Our first two equations are similar to LKE model, except that the phosphorus density of producer, $p$, and the free media phosphorus, $P$, are now explicitly modeled here. The above Assumption (A3) is no longer needed. The new model takes the form of

$$
\begin{aligned}
& \frac{\mathrm{d} x}{\mathrm{~d} t}=\underbrace{r x\left(1-\frac{x}{\min \{K, p / q\}}\right)}_{\text {producer growth limited by nutrient and light }}-\underbrace{f(x) y}_{\text {uptake by grazers }}, \\
& \frac{\underbrace{\mathrm{d} y}_{\text {grazer growth limited by food quality }}}{\mathrm{d} t}=\underbrace{\min \left\{1, \frac{p / x}{\theta}\right\} f(x) y}_{\text {grazer death and respiration loss }},
\end{aligned}
$$

$$
\frac{\mathrm{d} p}{\mathrm{~d} t}=\underbrace{g(P) x}_{\text {P uptake by producer }}-\underbrace{\frac{p}{x} f(x) y}_{\text {P loss due to grazing }}-\underbrace{\mathrm{d} p}_{\text {P loss due to producer recycling }},
$$

$$
\begin{array}{r}
\frac{\mathrm{d} P}{\mathrm{~d} t}=\underbrace{}_{\text {P uptake by producer P recycling }} \\
+\underbrace{\left(\frac{p}{x}-\hat{e} \min \left\{\theta, \frac{p}{x}\right\}\right) f(x) y .}_{\text {P recycling from grazer feces }} .
\end{array}
$$

The text below each term explains its biological meaning. $r$ is the intrinsic growth rate of the producer $\left(\mathrm{day}^{-1}\right), \hat{d}$ is the specific loss rate of the grazer that include the rates of respiration and death $\left(\right.$ day $\left.^{-1}\right) . d$ is the phosphorus loss rate representing $\mathrm{P}$ lost due to leave dropping, root 
decomposition and other recycling processes. $f(x)$ is the ingestion rate of the grazers, which will be assumed to follow Holling type II functional response in our analysis and simulation work. In general, $f(x)$ is a bounded smooth function that satisfies the following assumptions:

$$
f(0)=0, \quad f^{\prime}(x)>0 \text { and } f^{\prime \prime}(x) \leq 0, \quad \text { for } x \geq 0 .
$$

$g(P)$ is the phosphorus uptake rate of the producer. This function has similar properties as $f(x)$. $\hat{e}$ is the maximal production efficiency. The second law of thermodynamics requires that $\hat{e}<1$. $K$ represents a constant carrying capacity related to light. $q$ is the minimal cell quota $(\mathrm{P}: \mathrm{C})$ of the producer. $\theta$ is the fixed cell quota of the grazer. $\theta \gg q$ in reality; hence, we assume that $\theta>q$ in this paper.

In the free phosphorus equation, $g(P) x$ is the $\mathrm{P}$ uptake by the producer, $\mathrm{d} p$ and $\theta \hat{\mathrm{d}} y$ are $\mathrm{P}$ recycling from producer and consumer death,

$$
\left(\frac{p}{x}-\hat{e} \min \left\{\theta, \frac{p}{x}\right\}\right) f(x) y
$$

describes the consumer's $\mathrm{P}$ consumption from the producer minus the actual $\mathrm{P}$ retained due to growth and maintenance needs, which gives the amount of $\mathrm{P}$ recycled from consumer droppings and other losses.

$T=p+P+\theta y$ is the total phosphorus of the system, composed of producer phosphorus, free phosphorus and consumer phosphorus. By a simple calculation, we have $\mathrm{d} T / \mathrm{d} t=0$. The total phosphorus of System (1) is kept at the constant level of $T(0)=p(0)+P(0)+\theta y(0)$. Thus, $P \equiv T-p-\theta y$, which can be used to reduce System (1) by one dimension to

$$
\begin{aligned}
& \frac{\mathrm{d} x}{\mathrm{~d} t}=r x\left(1-\frac{x}{\min \{K, p / q\}}\right)-f(x) y, \\
& \frac{\mathrm{d} y}{\mathrm{~d} t}=\hat{e} \min \left\{1, \frac{p / x}{\theta}\right\} f(x) y-\hat{\mathrm{d}} y, \\
& \frac{\mathrm{d} p}{\mathrm{~d} t}=g(T-p-\theta y) x-\frac{p}{x} f(x) y-\mathrm{d} p .
\end{aligned}
$$

\begin{tabular}{|c|c|c|c|}
\hline $\mathrm{P}$ & Description & $\mathrm{V}$ & Unit \\
\hline$r$ & Intrinsic growth rate of the resource & 0.93 & day $^{-1}$ \\
\hline$K$ & Resource carrying capacity determined by light & $0.25-2$ & $(\mathrm{mg} \mathrm{C}) / 1$ \\
\hline$c$ & Maximal ingestion rate of the grazer & 0.75 & day $^{-1}$ \\
\hline$\hat{c}$ & Maximal phosphorus uptake rate of the producer & 0.2 & $(\mathrm{mg} \mathrm{P}) /(\mathrm{mg} \mathrm{C}) /$ day \\
\hline$a$ & Half-saturation constant of the grazer & 0.25 & $(\mathrm{mg} \mathrm{C}) / 1$ \\
\hline$\hat{a}$ & Phosphorus half-saturation constant of the producer & 0.008 & $(\mathrm{mg} \mathrm{P}) / 1$ \\
\hline$\hat{e}$ & Maximal conversion rate of the grazer & 0.74 & \\
\hline$\hat{d}$ & Loss rate of the grazer & 0.22 & day $^{-1}$ \\
\hline$d$ & Phosphorus loss rate of the producer & 0.05 & day $^{-1}$ \\
\hline$\theta$ & Constant $\mathrm{P}: \mathrm{C}$ of the grazer & 0.04 & $(\mathrm{mg} \mathrm{P}) /(\mathrm{mg} \mathrm{C})$ \\
\hline$q$ & Minimal possible $\mathrm{P}: \mathrm{C}$ of the producer & 0.004 & $(\mathrm{mg} \mathrm{P}) /(\mathrm{mg} \mathrm{C})$ \\
\hline$T$ & Total phosphorus in the system & 0.03 & $(\mathrm{mg} \mathrm{P}) / 1$ \\
\hline
\end{tabular}

The parameters in the $\mathbf{P}$ column of Table 1 will be needed for our subsequent study of System (3). Their values in the $\mathbf{V}$ column will be used for numerical simulations to be present later.

Table 1. The parameters (P) of System (3) and their values (V) used for numerical simulations. 


\section{Mathematical analysis}

In order to have a basic mathematical understanding of Model (3), we would like to study the dissipativity of the system and the stability of its boundary steady states. The following theorem shows that the system is naturally dissipative, meaning that the realistic solutions will remain in a biologically meaningful region.

\section{THEOREM 3.1 Solutions with initial conditions in the set}

$$
\Omega=\{(x, y, p): 0<x<\min \{K, T / q\}, 0<y, 0<p, p+\theta y<T\}
$$

remain there for all forward times.

Proof We consider a solution $X(t) \equiv(x(t), y(t), p(t)$ of (3) with initial condition in $\Omega$. Hence, $0<x(0)<\min \{K, T / q\}, 0<y(0), 0<p(0), p(0)+\theta y(0)<T$. Assume that there is time $t_{1}>0$ such that $X(t)$ touches or crosses the boundary of $\bar{\Omega}$ (closure of $\Omega$ ) for the first time, and then $(x(t), y(t), p(t)) \in \Omega$ for $0 \leq t<t_{1}$. We will have several cases to consider.

Case $1 \quad x\left(t_{1}\right)=0$ but $p\left(t_{1}\right) \neq 0.0 \leq p(t), p(t)+\theta y(t) \leq T$ for $0 \leq t \leq t_{1}$ implies $y(t) \leq T / \theta$ for $0 \leq t \leq t_{1}$. Let $p_{1}=\min \left\{p(t): t \in\left[0, t_{1}\right]\right\}>0$. Then for $0 \leq t \leq t_{1}$, we have

$$
\begin{aligned}
\frac{\mathrm{d} x}{\mathrm{~d} t} & =r x\left(1-\frac{x}{\min \{K, p / q\}}\right)-f(x) y \\
& \geq r x\left(1-\frac{\min \{K, T / q\}}{\min \left\{K, p_{1} / q\right\}}\right)-f^{\prime}(0)(T / \theta) x \\
& =\left[r\left(1-\frac{\min \{K, T / q\}}{\min \left\{K, p_{1} / q\right\}}\right)-f^{\prime}(0)(T / \theta)\right] x \equiv \mu x,
\end{aligned}
$$

where $\mu$ is a constant. Then, $x(t) \geq x(0) \mathrm{e}^{\mu t}$ for $0 \leq t \leq t_{1}$, which implies that $x\left(t_{1}\right) \geq$ $x(0) \mathrm{e}^{\mu t_{1}}>0$, a contradiction.

Case $2 x\left(t_{1}\right)=\min \{K, T / q\} .0 \leq y(t), p(t)+\theta y(t) \leq T$ for $0 \leq t \leq t_{1}$ implies $p(t) \leq T$ for $0 \leq t \leq t_{1}$. Then for $0 \leq t \leq t_{1}$, we have

$$
\frac{\mathrm{d} x}{\mathrm{~d} t}=r x\left(1-\frac{x}{\min \{K, p / q\}}\right)-f(x) y \leq r x\left(1-\frac{x}{\min \{K, T / q\}}\right) .
$$

The standard comparison argument yields that $x(t)<\min \{K, T / q\}$ for all $0 \leq t \leq t_{1}$, a contradiction.

Case $3 y\left(t_{1}\right)=0$. Then for $0 \leq t \leq t_{1}$, we have

$$
\frac{\mathrm{d} y}{\mathrm{~d} t}=\hat{e} \min \left\{1, \frac{p / x}{\theta}\right\} f(x) y-\hat{\mathrm{d}} y \geq-\hat{\mathrm{d}} y .
$$

Hence, $y(t) \geq y(0) \mathrm{e}^{-\hat{\mathrm{d}} t}>0$ for $0 \leq t \leq t_{1}$, a contradiction. 
Case $4 \quad p\left(t_{1}\right)=0 . \quad p(t)+\theta y(t) \leq T$ for $0 \leq t \leq t_{1}$ implies $T-p(t)-\theta y(t) \geq 0$ for $0 \leq t \leq t_{1}$. Thus, $g(T-p(t)-\theta y(t)) \geq 0$ for $0 \leq t \leq t_{1}$. Since $0 \leq p(t), p(t)+\theta y(t) \leq T$ for $0 \leq t \leq t_{1}$, then $y(t) \leq T / \theta$ for $0 \leq t \leq t_{1}$. Thus, for $0 \leq t<t_{1}$, we have

$$
\frac{\mathrm{d} p}{\mathrm{~d} t}=g(T-p-\theta y) x-\frac{p}{x} f(x) y-d p \geq-\frac{p}{x} f(x) y-d p \geq\left[-f^{\prime}(0)(T / \theta)-d\right] p \equiv v p,
$$

where $v$ is a constant. Thus, $p(t) \geq p(0) \mathrm{e}^{v t}>0$ for $0 \leq t \leq t_{1}$, a contradiction.

Case $5 p\left(t_{1}\right)+\theta y\left(t_{1}\right)=T$. Let $z(t)=T-p(t)-\theta y(t)$, then $z\left(t_{1}\right)=0$ and $z(t)>0$ for $0 \leq t<t_{1}$. Then for $0 \leq t \leq t_{1}$, we have

$$
\begin{aligned}
\frac{\mathrm{d} z}{\mathrm{~d} t} & =-\frac{\mathrm{d} p}{\mathrm{~d} t}-\theta \frac{\mathrm{d} y}{\mathrm{~d} t} \geq-g(z) x+d p+\hat{d} \theta y \\
& \geq-g^{\prime}(0) z \min \{K, T / q\}+\min \{d, \hat{d}\}(T-z) \\
& =\min \{d, \hat{d}\} T-\left[g^{\prime}(0) \min \{K, T / q\}+\min \{d, \hat{d}\}\right] z \equiv \tilde{\mu}-\tilde{v} z,
\end{aligned}
$$

where $\tilde{\mu}>0$ and $\tilde{v}>0$ are constant. Thus, $z(t) \geq \mathrm{e}^{-\tilde{v} t} z(0)>0$ for $0 \leq t \leq t_{1}$, a contradiction.

The stability analysis for general forms of $f(x)$ and $g(P)$ is tedious. For simplicity, we assume below that $g(P)=\alpha P$ and $f(x)=\beta x$. In this special case, Model (3) takes the following explicit form system becomes

$$
\begin{aligned}
\frac{\mathrm{d} x}{\mathrm{~d} t} & =r x\left(1-\frac{x}{\min \{K, p / q\}}\right)-\beta x y \equiv x F(x, y, p), \\
\frac{\mathrm{d} y}{\mathrm{~d} t} & =\hat{e} \min \left\{1, \frac{p / x}{\theta}\right\} \beta x y-\hat{d} y \equiv y G(x, y, p), \\
\frac{\mathrm{d} p}{\mathrm{~d} t} & =\alpha(T-p-\theta y) x-\beta p y-d p \equiv H(x, y, p) .
\end{aligned}
$$

The boundary equilibria include the total extinction equilibrium $E_{0}=(0,0,0)$ and the grazer extinction equilibrium

$$
E_{1}= \begin{cases}\left(K, 0, \frac{T K}{K+d / \alpha}\right), & \text { if } K<\frac{T}{q}-\frac{d}{\alpha} \\ \left(\frac{T}{q}-\frac{d}{\alpha}, 0, q\left(\frac{T}{q}-\frac{d}{\alpha}\right)\right), & \text { if } K>\frac{T}{q}-\frac{d}{\alpha},\end{cases}
$$
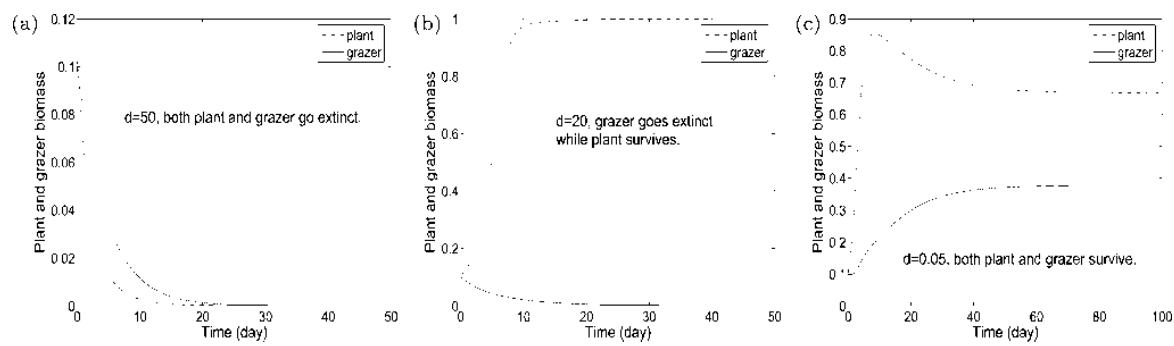

Figure 1. Figure illustration of Theorems 3.2-3.5 by choosing different $d$ values. (a) The extinction steady state $E_{0}$ is asymptotically stable.(b) The grazer extinction steady state $E_{1}$ is asymptotically stable. (c) The coexistence steady state $E^{*}$ is asymptotically stable. 
if $T / q-d / \alpha>0$. Model (4) may also have a coexistence equilibrium $E^{*}$, which satisfies

$$
\begin{aligned}
r\left(1-\frac{x}{\min \{K, p / q\}}\right) & =\beta y, \\
\hat{e} \min \left\{1, \frac{p / x}{\theta}\right\} \beta x & =\hat{d}, \\
\alpha(T-p-\theta y) x & =p \beta y+d p .
\end{aligned}
$$

For the extinction steady state $E_{0}$, we have the following theorem for the general system (3).

THEOREM 3.2 The extinction steady-state $E_{0}=(0,0,0)$ in System (3) (or (4)) is globally asymptotically stable if $d>m g(T)$, where $m=\min \left\{x(0) / p(0), 1+\left[\left(d+f^{\prime}(0) T / \theta\right) / r\right] / q\right\}$.

Proof Let $u=x / p$, then

$$
\begin{aligned}
\frac{\mathrm{d} u}{\mathrm{~d} t} & =\frac{\mathrm{d} x / \mathrm{d} t}{p}-u \frac{\mathrm{d} p / \mathrm{d} t}{p} \\
& \leq r u(1-q u)+\left(d+f^{\prime}(0) y\right) u \\
& \leq r u\left(1+\left(d+f^{\prime}(0) y\right) / r-q u\right) \\
& \leq r u\left(1+\left(d+f^{\prime}(0) T / \theta\right) / r-q u\right) .
\end{aligned}
$$

Hence, $u \leq \min \left\{x(0) / p(0),\left[1+\left(d+f^{\prime}(0) T / \theta\right) / r\right] / q\right\} \equiv m$. For the $p$ equation, $\mathrm{d} p / \mathrm{d} t \leq$ $g(T) x-\mathrm{d} p \leq g(T) m p-\mathrm{d} p=(g(T) m-d) p$. Since $d>m g(T)$ implies $g(T) m-d<0$, hence $p \rightarrow 0$ as $t \rightarrow \infty$.

Next, we show that $x \rightarrow 0$ as $t \rightarrow \infty$. This can be seen from the fact that $\mathrm{d} x / \mathrm{d} t \leq r x$ $\left(1-(q x / p)\right.$, which implies that $\lim \sup _{t \rightarrow \infty} x(t) \leq p / q$. Clearly, this also implies that $y \rightarrow 0$ as $t \rightarrow \infty$.

The above theorem states that both the plant and the grazer go extinct when the nutrient loss rate is high. Plant extinction cannot be obtained in most previous models including the LKE model, except in pure ratio-dependent models [6]. Therefore, our model provides an alternative and possibly less controversial deterministic mechanism to exhibit such total and realistic extinction dynamics.

We will compute the Jacobian matrix through $F, G, H$ and their partial derivatives. The partial derivatives of these functions are

$$
\begin{array}{rlrl}
F_{x} & =-\frac{r}{\min \{K, p / q\}}, & F_{y}=-\beta, & F_{p}= \begin{cases}0 & \text { if } p>q K, \\
\frac{r q x}{p^{2}} & \text { if } p<q K,\end{cases} \\
G_{x}= \begin{cases}\hat{e} \beta \text { if } p>\theta x, \\
0 \quad \text { if } p<\theta x,\end{cases} & G_{y}=0, & G_{p}= \begin{cases}0 & \text { if } p>\theta x, \\
\frac{\hat{e} \beta}{\theta} & \text { if } p<\theta x,\end{cases} \\
H_{x} & =\alpha(T-p-\theta y), & H_{y}=-\alpha \theta x-\beta p, & H_{p}=-\alpha x-\beta y-d .
\end{array}
$$

The Jacobian matrix is

$$
A=\left(\begin{array}{ccc}
F+x F_{x} & x F_{y} & x F_{p} \\
y G_{x} & G+y G_{y} & y G_{p} \\
H_{x} & H_{y} & H_{p}
\end{array}\right) .
$$

The trace of $A, \operatorname{tr} A=F+x F_{x}+G+y G_{y}+H_{p}$. 
The determinant of $A, \operatorname{det} A=\left(F+x F_{x}\right)\left(G+y G_{y}\right) H_{p}+x y F_{y} G_{p} H_{x}+x y F_{p} G_{x} H_{y}-$ $x F_{p}\left(G+y G_{y}\right) H_{x}-x y F_{y} G_{x} H_{p}-\left(F+x F_{x}\right) y G_{p} H_{y}=x H_{x}\left(y F_{y} G_{p}-F_{p} G-y F_{p} G_{y}\right)+$ $y H_{y}\left(x F_{p} G_{x}-F G_{p}-x F_{x} G_{p}\right)+H_{p}\left(F G+y F G_{y}+x F_{x} G+x y F_{x} G_{y}-x y F_{y} G_{x}\right)$.

Let $A_{j k}$ be the determinant of the $2 \times 2$ matrix resulted from A by removing the $j$ th row and the $k$ th column. Then, $A_{11}=\left(G+y G_{y}\right) H_{p}-y G_{p} H_{y}, A_{22}=\left(F+x F_{x}\right) H_{p}-x F_{p} H_{x}, A_{33}=$ $\left(F+x F_{x}\right)\left(G+y G_{y}\right)-x y F_{y} G_{x}$.

By Routh-Hurwitz criterion, all eigenvalues of $A$ have strictly negative real parts if the following conditions hold.

(i) $\operatorname{tr} A<0$; $\quad$ (ii) $\operatorname{det} A<0 ; \quad$ (iii) $\operatorname{det} A-(\operatorname{tr} A)\left(\sum_{k=1}^{3} A_{k k}\right)>0$.

The following theorem presents a sufficient condition for the local stability of $E_{1}$ when $K>$ $T / q-d / \alpha>0$.

THEOREM 3.3 For the case of $K>T / q-d / \alpha>0, E_{1}$ is locally asymptotically stable if $\hat{e} q \beta / \theta((T / q)-(d / \alpha))<\hat{d}$.

Proof Recall that $E_{1}=(\bar{x}, 0, \bar{p})$ where $\bar{x}=(T / q)-(d / \alpha), \bar{p}=q((T / q)-(d / \alpha))$. Clearly, $\bar{x}<K, \bar{p}<q K$. Since $\theta>q$, we have $\bar{p}<\theta \bar{x}$. Observe that $U=(\hat{e} q \beta / \theta) \bar{x}-\hat{d}<0$, $V=(\alpha \operatorname{Tr} / q)-r d>0$ and $W=\alpha \bar{x}+r+d>0$ by conditions of the theorem and the positivity of parameters. In our case, the Jacobian matrix at $E_{1}$ becomes

$$
A=\left(\begin{array}{ccc}
-r & -\beta \bar{x} & r / q \\
0 & U & 0 \\
\alpha(T-\bar{p}) & -\alpha \theta \bar{x}-\beta \bar{p} & -\alpha \bar{x}-d
\end{array}\right) .
$$

The $U, V$ and $W$ formulae together with some straightforward calculations yield

$$
\operatorname{tr} A=U-\alpha \bar{x}-r-d<0, \quad \operatorname{det} A=U V<0,
$$

and

$$
\operatorname{det} A-(\operatorname{tr} A)\left(\sum_{k=1}^{3} A_{k k}\right)=W\left(U^{2}-U W+V\right)>0 .
$$

The theorem follows from an application of the Routh-Hurwitz criterion.

The next theorem presents a sufficient condition for the local stability of $E_{1}$ when $K<T / q-d / \alpha>0$ and $T<\theta(K+d / \alpha)$.

THEOREM 3.4 Assume that $K<(T / q)-(d / \alpha)$ and $T /(K+d / \alpha)<\theta$. Then, $E_{1}$ is locally asymptotically stable if $(\hat{e} \beta / \theta)(T K / K+(d / \alpha))<\hat{d}$.

Proof In this case, $E_{1}=(\bar{x}, 0, \bar{p})$ where $\bar{x}=K, \bar{p}=T K /(K+(d / \alpha))$. We see that $\bar{p}>q K, \bar{p}<\theta \bar{x}$. Observe that $U=(\hat{e} \beta \bar{p} / \theta)-\hat{d}=(\hat{e} \beta / \theta)(T K / K+(d / \alpha))-\hat{d}<0$ and $V=-\alpha \bar{x}-d<0$. By straightforward calculations involving the Jacobian matrix,

$$
A=\left(\begin{array}{ccc}
-r & -\beta K & 0 \\
0 & U & 0 \\
\alpha(T-\bar{p}) & -\alpha \theta \bar{x}-\beta \bar{p} & -\alpha \bar{x}-d
\end{array}\right) .
$$

we have $\operatorname{tr} A=U+V-r<0, \operatorname{det} A=-r U V<0$ and

$$
\operatorname{det} A-(\operatorname{tr} A)\left(\sum_{k=1}^{3} A_{k k}\right)=-(U+V)(U V-r U-r V)-r^{2} U-r^{2} V>0 .
$$

The theorem follows from an application of the Routh-Hurwitz criterion. 
Table 2. Stability results of $E_{1}$ in System (4).

\begin{tabular}{lcl}
\hline Case & Condition & Stability \\
\hline$\frac{T}{q}-\frac{d}{\alpha} \leq 0$ & Not exist \\
$K>\frac{T}{q}-\frac{d}{\alpha}>0$ & $\frac{\hat{e} q \beta}{\theta}\left(\frac{T}{q}-\frac{d}{\alpha}\right)<\hat{d}$ & LAS \\
$\frac{\hat{e} q \beta}{\theta}\left(\frac{T}{q}-\frac{d}{\alpha}\right)>\hat{d}$ & Unstable \\
$\frac{T}{\theta}-\frac{d}{\alpha}<K<\frac{T}{q}-\frac{d}{\alpha}$ & $\frac{\hat{e} \beta}{\theta} \frac{T K}{K+d / \alpha}<\hat{d}$ & LAS \\
$\frac{\hat{e} \beta}{\theta} \frac{T K}{K+d / \alpha}>\hat{d}$ & Unstable \\
& $\hat{e} \beta K<\hat{d}$ & LAS (Locally asymptotically stable) \\
\hline
\end{tabular}

Our last theorem presents a sufficient condition for the local stability of $E_{1}$ when $K<T / q-d / \alpha>0$ and $T>\theta(K+d / \alpha)$.

THEOREM 3.5 Assume that $K<(T / q)-(d / \alpha)$ and $(T / K+(d / \alpha))>\theta$. Then, $E_{1}$ is locally asymptotically stable if $\hat{e} \beta K<\hat{d}$.

Proof We see that $E_{1}=(\bar{x}, 0, \bar{p})$ where $\bar{x}=K, \bar{p}=T K /(K+d / \alpha)$. Then, $\bar{p}>q K, \bar{p}>\theta \bar{x}$. Let $U=\hat{e} \beta \bar{x}-\hat{d}=\hat{e} \beta K-\hat{d}<0$ and $V=-\alpha \bar{x}-d<0$. By straightforward calculations, we see that the Jacobian matrix in this case is the same as that in the previous theorem with the new definition of $U$. The rest of the proof is identical to that of the previous theorem.

Observe that when $(T / q)-(d / \alpha) \leq 0, E_{1}$ does not exist. $E_{1}$ degenerates to $E_{0}$ if $(T / q)-$ $(d / \alpha)=0$. Instability results of $E_{1}$ can be easily obtained from the conditions lead to $\operatorname{det} A>0$. In addition, $\theta>q$ is biologically reasonable. With this assumption, the condition ' $K<(T / q)-$ $(d / \alpha)$ and $(T /(K+(d / \alpha))<\theta$ ' is equivalent to ' $(T / \theta)-(d / \alpha)<K<(T / q)-(d / \alpha)$ ', and the condition ' $K<(T / q)-(d / \alpha)$ and $(T / K+(d / \alpha))>\theta$ ' is equivalent to ' $K<(T / \theta)-$ $(d / \alpha)^{\prime}$. We summarize these results on $E_{1}$ in Table 2 . All stability results in this section are illustrated in Figure 1.

\section{Numerical dynamics and its implications}

The initial conditions in our simulation are chosen inside the biologically meaningful region $\Omega$. We let $f(x)=c x /(a+x)$ and $g(P)=\hat{c} P /(\hat{a}+P)$ in our simulations.

Since the carrying capacity $K$ can be linked to or regarded as the light intensity in lab and field experiments, it is one of our natural bifurcation parameters. In Figure 2, We vary the carrying capacity $K$ in the bifurcation diagrams. Other parameter values are given in Table 1 . In this figure, we simulate Model (3) with faster nutrient process and compare the results to those of LKE model. In Figure 2, as $K$ increases, the stable positive steady-state loses its stability through a Hopf bifurcation point and gives rise to a limit cycle. Further increasing $K$ collapses the cyclic behaviour through a homoclinic bifurcation and returns the dynamics to a stable steady-state 
(a)

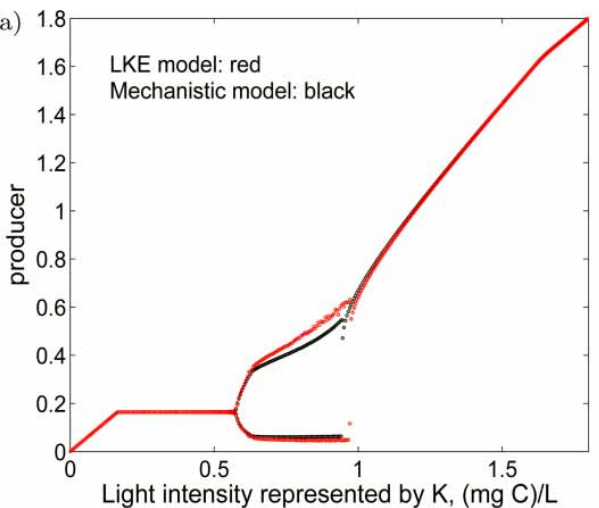

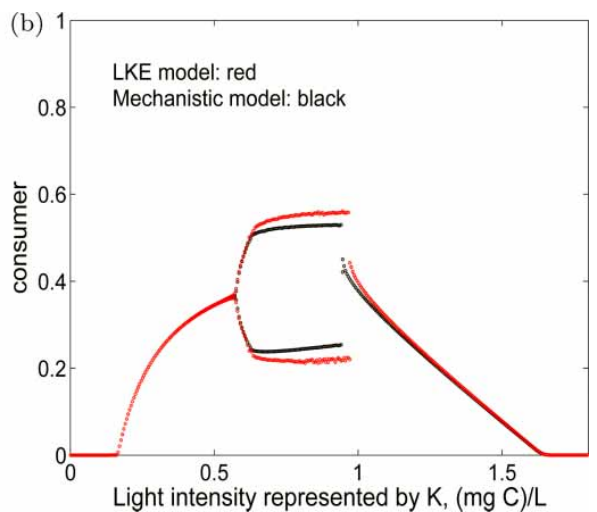

Figure 2. Bifurcation diagrams for Model (3) with respect to $K$. (a) producer vs. $K$; (b) consumer $v s . K$.

behavior. The consumer goes extinct for extremely small $K$ because of low food quantity, or for extremely large $K$ because of the low food quality (panel (b) in Figure 2). Solutions of Model (3) are compared to that of the LKE model for various $K$ values in Figure 3. For small or large $K$ (steady-state cases, panels (a) and (d) in Figure 3), our model and the LKE model have almost identical solutions. For the intermediate $K$ values (panels (b) and (c) in Figure 3), they are slightly different quantitatively. However, when $K$ is near the homoclinic bifurcation point (see Figure 4), they are completely different. Near this point, the solution sensitivity with respect to $K$ is very high. Nevertheless, in general, we can state that (see the bifurcation diagrams (Figure 2)) the LKE model is a very good approximation of the mechanistic Model (3).
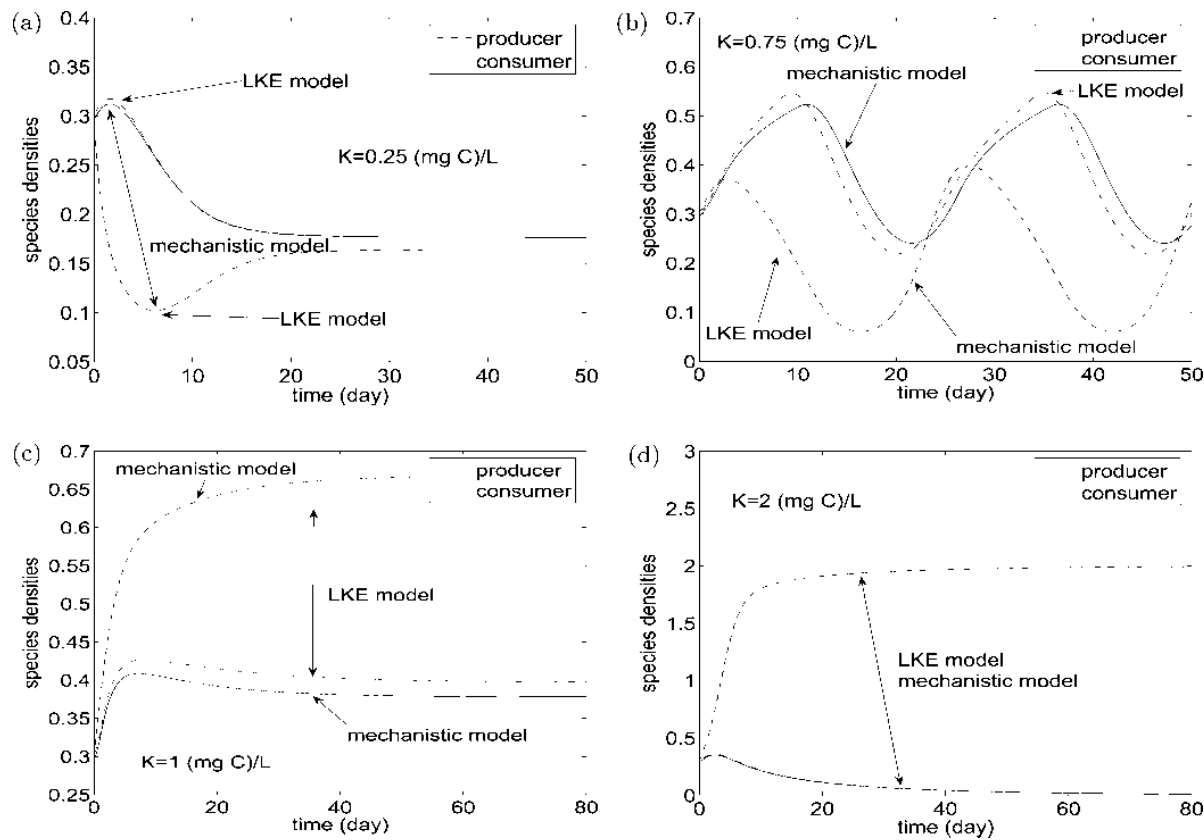

Figure 3. Comparison of solutions of the mechanistic model and the LKE model with different carrying capacities. (a) coexistence at a steady state; (b) coexistence with oscillations; (c) coexistence at a steady state with a higher producer/grazer ratio; (d) extinction of the grazer. 


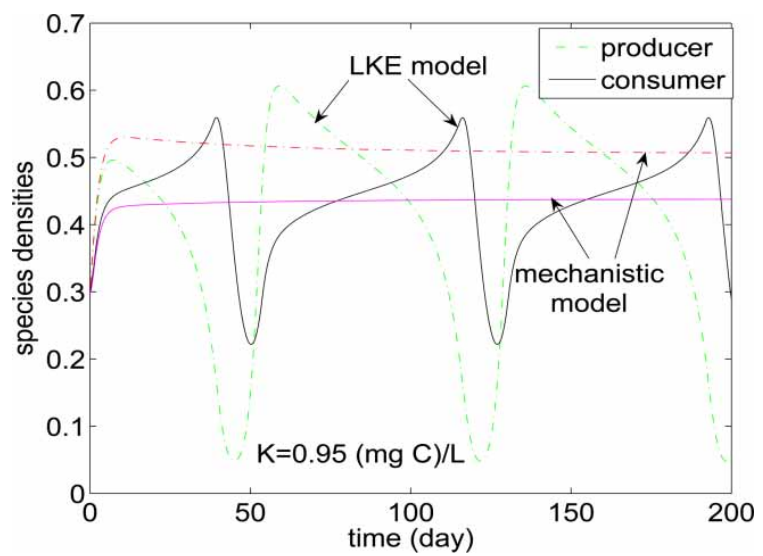

Figure 4. Comparison of Model (3) with the LKE model with the following parameter values: $r=0.93, c=0.75$, $\hat{c}=0.2, a=0.25, \hat{a}=0.008, e=0.74, d=0.22, \hat{d}=0.05, \theta=0.04, q=0.004, T=0.03$.

\section{Discussion}

We constructed and analyzed a stoichiometric predator-prey model that explicitly tracks the quantity and the nutritional quality of the prey. We achieve this by mechanistically accounting for the content of two essential elements in this simple food web, C and P. Carbon is considered to be energy (quantity) equivalent, while the concentration of $\mathrm{P}$ (or $\mathrm{P}: \mathrm{C}$ ratio) reflects nutritional quality of the prey. This model builds upon the LKE model by expanding it from a two-dimensional system to a four-dimensional one. The additional two equations track $\mathrm{P}$ in the prey and in the media that supports the prey. The LKE model assumes that the prey is extremely efficient at uptaking nutrient (P) from the media; hence, Assumption (A3), no P in the media. Interestingly, if we assume in Model (4) the infinite uptake efficiency of prey $(\alpha \rightarrow \infty)$, then our model converges naturally to the LKE model. Here is the argument. Observe that the nutrient $p$ in the producer and $P$ in the media possess a dynamics that is much faster than growth dynamics of producer and consumer. We claim that Model (4) has almost the same dynamics as that of the LKE model. This can be seen by a simple application of the quasi-steady-state argument on the nutrient equation (4c). This yields $p$ as a function of $x$ and $y$, which is $\alpha x(T-\theta y) /(\alpha x+\beta y+d)$. Thus, Equation (4) can be reduced to

$$
\begin{aligned}
& \frac{\mathrm{d} x}{\mathrm{~d} t}=r x\left(1-\frac{x}{\min \{K,(\alpha x(T-\theta y) / \alpha x+\beta y+d) / q\}}\right)-\beta x y, \\
& \frac{\mathrm{d} y}{\mathrm{~d} t}=\hat{e} \min \left\{1, \frac{[\alpha x(T-\theta y) / \alpha x+\beta y+d] / x}{\theta}\right\} \beta x y-\hat{d} y .
\end{aligned}
$$

The plant phosphorus in the LKE model is $T-\theta y$, a function of $y$, following Assumption (A3). The phosphorus outside the grazer, $T-\theta y$, is distributed between the plant and the media, and the plant phosphorus has the fraction $\alpha x /(\alpha x+\beta y+d)$. Therefore, the plant phosphorus should be a function of both $x$ and $y$. $\alpha$ is large in fast nutrient process, and $\alpha x /(\alpha x+\beta y+d) \approx 1$ for sufficiently large $\alpha$; then, the plant phosphorus is almost $T-\theta y$ for fast nutrient process. Hence, the LKE model can be regarded as the limiting case of Model (4) when $\alpha \rightarrow \infty$.

However, even with finite nutrient uptake rates for the producer, our model behaves both qualitatively and quantitatively similar to the LKE model as bifurcation diagrams (2) and simulations (3) show. The exception is the interval for $K$ near the point where the predator limitation switches from $\mathrm{C}$ to $\mathrm{P}$ (from food quantity to food quality), where there is a quantitative difference between the two models. This raises the question whether the significant similarity between the two models justifies the additional complexity of our model. We certainly think so for the following reasons. 
In applications, such as the control of eutrophication in lakes, it is the actual concentration of $\mathrm{P}$ in the water that is of crucial importance, and our model explicitly tracks it. This mechanistical model formulation also provides an additional significant benefit; our model can be easily expanded to multiple producers and grazers while maintaining its structure. This later property is not shared by the LKE model because Assumption (A3) does not leave a mechanistic way to distribute $\mathrm{P}$ among multiple producers.

\section{Acknowledgements}

The research of Yang Kuang and Hao Wang is supported in part by DMS-0436341 and DMS/NIGMS-0342388. Correspondence should be directed to Hao Wang.

\section{References}

[1] T. Andersen, Pelagic Nutrient Cycles: Herbivores as Sourced and Sinks for Nutrients. Springer-Verlag, Berlin, 1997.

[2] T. Andersen, J.J. Elser, and D.O. Hessen, Stoichiometry and population dynamics, Ecol. Lett. 7 (2004) pp. 884-900.

[3] J.J. Elser, et al., Nutritional constraints in terrestrial and freshwater food webs, Nature 408 (2000) pp. 578-580.

[4] J.P. Grover, Stoichiometry, herbivory and competition for nutrients: Simple models based on planktonic ecosystems, J. Theor. Biol. 214 (2002) pp. 599-618.

[5] D.O. Hessen and B. Bjerkeng, A model approach to planktonic stoichiometry and consumerCresource stability, Freshwater Biol. 38 (1997) pp. 447-471.

[6] Y. Kuang and E. Beretta, Global qualitative analysis of a ratio-dependent predator-prey system, J. Math. Biol. 36 (1998) pp. 389-406.

[7] Y. Kuang, J. Huisman, and J.J. Elser, Stoichiometric plant-herbivore models and their interpretation, Math. Biosc. Eng. 1 (2004) pp. 215-222.

[8] I. Loladze, Y. Kuang, and J.J. Elser, Stoichiometry in producer-grazer systems: Linking energy flow with element cycling, Bull. Math. Biol. 62 (2000) pp. 1137-1162.

[9] I. Loladze, et al., Competition and stoichiometry: Coexistence of two predators on one prey, Theo. Popu. Biol. 65 (2004) pp. 1-15.

[10] E.B. Muller, et al. Stoichiometric food quality and herbivore dynamics, Ecol. Lett. 4 (2004) pp. 519-529.

[11] R.W. Sterner and J.J. Elser, Ecological Stoichiometry, Princeton University, Princeton, NJ, 2002. 